\title{
Robust power allocation for two-tier heterogeneous networks under channel uncertainties
}

\author{
Yongjun $\mathrm{Xu}^{1,2^{*}+}$, Xiaolei $\mathrm{Yu}^{1+}$, Yuchao $\mathrm{Liu}^{1}$ and Guoquan $\mathrm{Li}^{1}$
}

\begin{abstract}
In this paper, the trade-off among system sum energy consumption and robustness is studied. In this regard, a robust power allocation problem is formulated for a two-tier heterogeneous network with uplink transmission mode and consideration of imperfect channel state information. The objective is to minimize the total transmit power of femtocell users (FUs), while the interference to macrocell user receiver is limited to a predefined interference level, the transmit power of each FU transmitter is kept within their power budgets, and the actual signal-to-interference-plus-noise ratio of each femtocell receiver is above a minimum threshold. Considering the uncertainties of the interference links from FUs to macrocell base stations and forward transmission links of each FU, the robust power allocation problem is formulated as a semi-infinite programming problem (SIPP). By the worst-case approach, the SIPP is transformed into a convex optimization problem solved by the Lagrange dual decomposition method. Moreover, the feasible regions of constraints, computational complexity, and sensitivity degree of the proposed robust algorithm are also analyzed. Simulation results investigate the impact of channel uncertainties and the superiority of the proposed algorithm by comparing with non-robust algorithm.
\end{abstract}

Keywords: Heterogenous networks, Robust power allocation, Channel uncertainty, Worst-case approach

\section{Introduction}

With the rapid increase of mobile data, more than $50 \%$ phone calls and $70 \%$ data services take place in indoor environment [1]. However, traditional homogeneous cellular networks cannot meet this requirement. Femtocell enabled in macrocell networks consists of a new heterogeneous cellular network which can satisfy the requirement of the increasing wireless data services due to low-power consumption and flexible deployment of femtocell users [2]. In HetNets, there are usually two types of users: FUs and MUs. On the one hand, FUs considered as low-power nodes utilize the same spectrum resource with MUs and improve indoor area coverage so that the spectrum efficiency and system capacity of communication system can be improved heavily. On the other hand, cross-tier

\footnotetext{
*Correspondence: xuyj@cqupt.edu.cn

${ }^{\dagger}$ Yongjun Xu and Xiaolei Yu contributed equally to this work.

${ }^{1}$ College of Communication and Information Engineering, Chongqing

University of Posts and Telecommunications, Chongqing 400065, China

${ }^{2}$ State Key Laboratory of Integrated Services Networks, Xidian University, Xi'an

710071, China
}

interference from femtocell networks to macrocell networks and the interference from MBS must be carefully controlled. Therefore, PA is a key technique for guaranteeing the QoS of users in HetNets.

Since PA can mitigate mutual interference of multiusers, ensure the QoS of each UE and improve system overall throughput, it has been considered as an effective method to achieve resource allocation in HetNets. In [3], for OFDMA femtocell networks, with consideration of FUs' fairness in each femtocell and protection of MUs, a PA algorithm is proposed via distributed FoschiniMiljanic power update technology. Similarly, in [4], a PA scheme with consideration of femtocell clustering is investigated based on branch-and-bound algorithm and the simplex algorithm to enhance data rate of FUs and alleviate the interference to MUs in macrocell-femtocell HetNets. A distributed utility-based SINR strategy for femtocell networks in [5] is investigated to reduce the cross-tier interference from femtocell networks to macrocell networks. But maximum transmission power limitation of each user is ignored in this paper. These PA 
schemes have an efficient performance in mitigating the interference between MUs and femtocell users under perfect CSI. However, in practical systems, perfect CSI is hard to be accurately acquired because of the effect of channel fading and feedback delays. Therefore, PA under imperfect CSI should be considered ahead of time in practical transmission system of HetNets.

Currently, to improve robustness of heterogeneous communication network, based on robust optimization theory, many authors have dedicated to study robust PA algorithms under channel uncertainty in two-tier HetNets [6]. In [7], to enhance the robustness of system, an uplink RPA problem is investigated in two-tier femtocell networks to deal with the uncertainties and protect the QoS of all users by using outage QoS constraints. Considering the same network scenario, a RPA scheme is put forward under channel uncertainties in [8] to maximize the network benefit among all users. In [9], a RPA algorithm is investigated to minimize the transmission power of FUs for energy-saving, which is a formulated subject to the QoS constraints and cross-tier interference constraints with the consideration of channel estimation errors. But the cross-tier interference received at FUs is ignored. Moreover, only single user scenario and the probability constraints are considered in [7-9]. A robust Stackelberg game is presented to formulate the two-tier uplink RPA problem to satisfy different service requirements of both FUs and MUs in [10]. However, they ignore SINR protection of FUs. To improve system capacity, in [11], a resource allocation scheme for two-tier OFDMbased cognitive femtocell networks is proposed by taking the mutual interference, imperfect spectrum sensing, and channel uncertainty into account, where the energy consumption is ignored. In [12], to maximize the utilities of all users, based on hierarchical game theory, the authors propose a robust uplink PA algorithm under the consideration of probability interference constraints. For a multitier cognitive HetNet, in [13], the authors study a SIP problem to maximize the SINR of microcell users under channel uncertainties, which is converted into a geometric programming problem by using a relaxation approach. In [14], based on worst-case theory, a distributed RPA algorithm is proposed to obtain maximum rate of femtocell users in OFDMA-based femtocell networks subject to intra-tier and cross-tier interference uncertainties. Aiming at enhancing the robustness of system, the author in [15] studies an outage-based robust optimization problem under partial CSI feedback and no CSI feedback. In [16], a RPA algorithm is proposed to minimize the total power of all users subject to outage probability constraints under time-varying wireless channels in two-tier femtocell networks. However, the existing works do not deal with the channel uncertainties with the consideration MU-to-FU links, interference links among FUs, and SINR requirement of FUs, simultaneously. Additionally, the feasible region of optimal power and sensitivity analysis is not considered.

Energy consumption, user performance, and robustness are the three important characteristics of each cellular network (i.e., macrocell network, femtocell network) in HetNets where the trade-off between optimality and robustness should be also studied. To this end, by considering the channel uncertainties in SINR constraint of each FU and interference power constraint to MUs, we investigate a RPA problem in two-tier HetNets under uplink transmission mode that minimizes the total transmit power of FUs. To solve the proposed problem, we transform the problem into a convex one by using bounded ellipsoidal model and worst-case approach, then the analytical solution is obtained by using Lagrange theory.

The main contributions of our paper are summarized as follows:

- We proposed a RPA algorithm based on energy minimization for the uplink of a HetNet with one macrocell and multiple femtocells by considering all channel uncertainties. Our motivations behind this system model are (a) multiple overlapped femtocell network is a more practical and promising candidate to improve system throughput and spectrum efficiency; (b) with considering all possible channel uncertainties, the robustness of system can be improved where both transmission links among different femtocells and transmission links in macrocell can be guaranteed at the same time.

- We used a simple method to transform the NP-hard problem into a convex one. Also the feasible regions of optimal PA problem and the proposed RPA problem are given.

- Then, we addressed the complexity and sensitivity degree of the algorithm and obtained the analytical relationship between overall energy consumption and uncertain parameters. The simulation parts demonstrated the effectiveness of the proposed algorithm.

The rest of the paper is given as follows. Section 2 presents the methods of this study. The system model is given in Section 3 and transformation process of the designed RPA problem is presented in Section 4. Section 5 proposes a RPA algorithm based on the above deterministic model. And the performance analysis is given in Section 6. The simulation results are presented in Section 7. Finally, the conclusion is given in Section 8.

\section{Methods}

Considering system energy consumption and transmission robustness of a HetNet with one macrocell with 
multiple femtocells, this study presented a power minimization scheme subject to all channel uncertainties. After network initialization is accomplished, our proposed RPA algorithm at each FU transmitter is used to adjust the corresponding transmit power to achieve total power consumption minimization under the constraints of interference power of MUs and SINR requirement of each FU. Due to instability of wireless channel, we considered all channel uncertainties and converted the nominal problem into a deterministic one based on worst-case principle. Then, the optimal solution can be obtained by utilizing Lagrange dual decomposition theory. The RPA algorithm can be accomplished by the following steps: (1) at FU's receiver, it estimates the forward channel gains and obtains the estimated direct channel gain values. Determine the error upper bound according to the robustness requirement of system and the accuracy of channel estimation algorithm. Then, the related system parameters (e.g., estimated channel gains, background noise power) are fed back to its transmitter. (2) Data fusion center at FU's BS collects the tolerable interference power levels, determines the minimum value, and broadcasts to all transmitters in femtocells. (3) Based on these system parameters and its own robustness requirement, each transmitter adjusts the transmit power by the designed RPA algorithm.

\section{System model}

We consider an uplink transmission model of two-tier HetNets with one macrocell and multiple femtocells as shown in Fig. 1 , where one MBS serves $L$ MUs and $K$ FBSs. Each FBS serves $M$ FUs. Define the set of FUs as $\forall i, j \in$ $\{1,2, \cdots, M\}$, the set of FBSs as $\forall k \in\{1,2, \cdots, K\}$, and the set of MUs as $\forall l \in\{1,2, \cdots, L\}$. We suppose that both users and femtocell base stations are randomly distributed in the coverage area. For the sake of clarity, Table 1 gives the summary of the notations which are adopted in this paper.

In HetNets, femtocells share the same frequency resources with macrocells [1]. To protect the basic QoS of MUs, we need to limit the interference power caused from femtocell networks to macrocell networks under a certain allowable range [17]. Therefore, we consider a global interference constraint at the FU side, i.e.,

$$
\sum_{k} \sum_{i} p_{i}^{k} G_{i}^{k} \leq I_{\mathrm{th}}
$$

According to information theory, the received SINR at FBS over link $i$ can be formulated as

$$
\gamma_{i}^{k}=\frac{p_{i}^{k} h_{i}^{k}}{\sum_{j \neq i}^{M} p_{j}^{k} h_{j}^{k}+\sum_{l=1}^{L} p_{l} g_{l}^{k}+\sigma_{i}^{k}},
$$
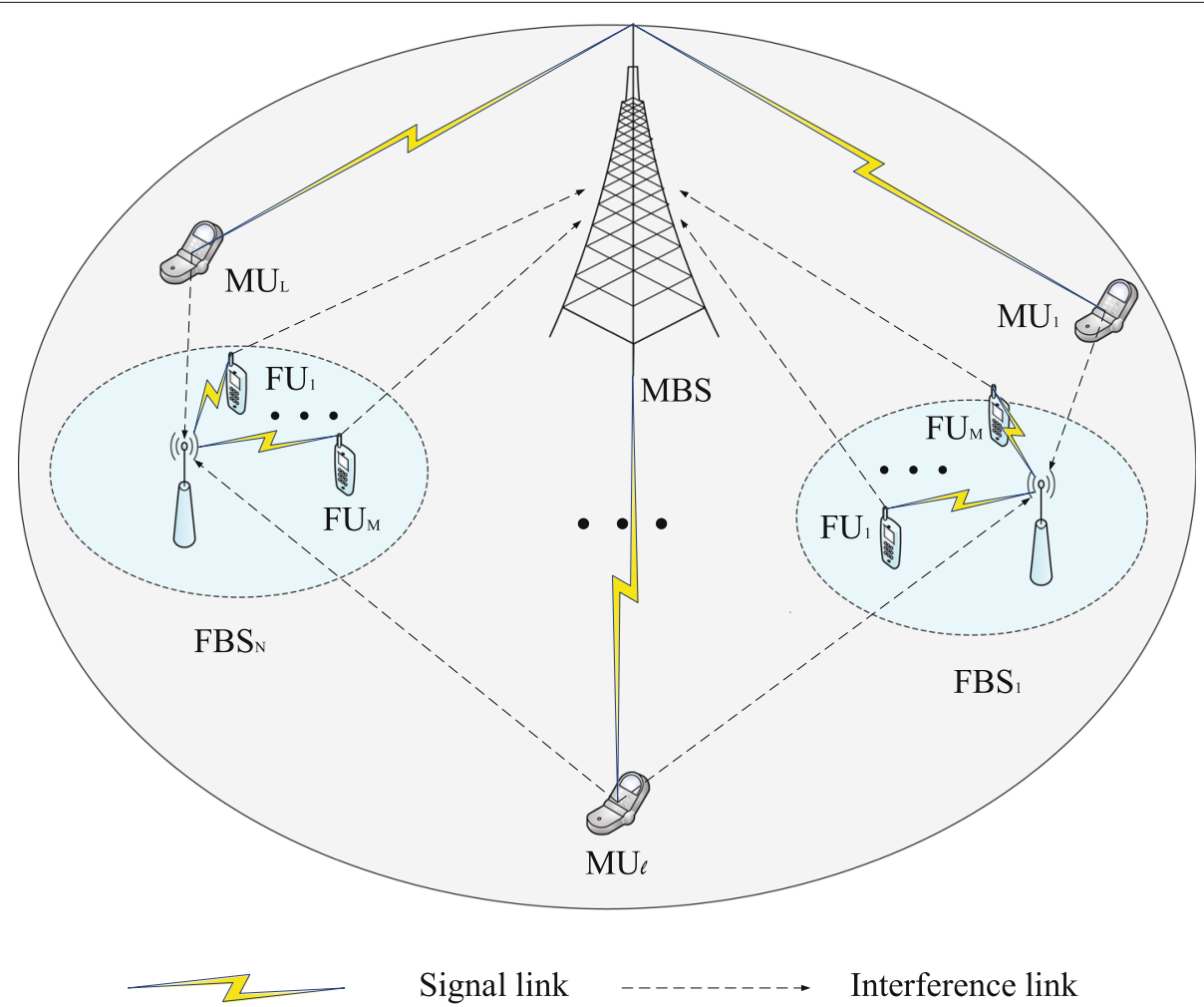

Signal link

Interference link

Fig. 1 System model. Signal link and interference link 
Table 1 Symbol definition

\begin{tabular}{ll}
\hline Notation & Description \\
\hline$G_{i}^{k}$ & $\begin{array}{l}\text { Channel gain between the ith FU of the femtocell network } k \\
\text { and MBS. }\end{array}$ \\
$p_{i}^{k}$ & $\begin{array}{l}\text { transmit power of the } i \text { th FU in the femtocell network } k . \\
l_{t h}\end{array}$ \\
$\gamma_{i}^{k}$ & Maximum interference power that the MBS can tolerate. \\
$h_{i}^{k}$ & Channel gain between $i$ th FU-Tx and $k$ th FBS. \\
$h_{j}^{k}$ & Channel gain between $j$ th FU-Tx and $k$ th FBS. \\
$g_{i}^{k}$ & Interference channel gain from the $/$ th MU-Tx to the $k$ th FBS. \\
$p_{l}$ & Transmit power of the $/$ th MU. \\
$\sigma_{i}^{k}$ & Background noise received at each FBS. \\
\hline
\end{tabular}

where the first term of denominator denotes the interference power from neighboring FUs (i.e., intra-tier interference). The second part of denominator is the interference power from macrocell networks (i.e., cross-tier interference).

To guarantee transmission qualities of each FU, i.e., the received SINR at each FBS (i.e., $\gamma_{i}^{k}$ ) should be bigger than a minimum SINR threshold, which is given as

$$
\gamma_{i}^{k} \geq \gamma_{i}^{k, \min }
$$

where $\gamma_{i}^{k \text {,min }}$ denotes the minimum SINR of the $i$ th FU in the $k$ th femtocell.

Considering the limitation of battery capacity of FUs, the transmit power of each FU is bounded, and we have the following constraint,

$$
0 \leq p_{i}^{k} \leq p_{i}^{k, \max }
$$

where $p_{i}^{k, \max }$ denotes the maximum transmission power of the $i$ th FU in femtocell network $k$.

In order to better analyze the impact of interference from femtocells to macrocells, we define the outage probability of MUs as follows,

$$
P(m)= \begin{cases}0, & I_{\mathrm{ac}}<I_{\mathrm{th}} \\ \frac{I_{\mathrm{ac}}-I_{\mathrm{th}}}{I_{\mathrm{th}}}, & I_{\mathrm{ac}} \geq I_{\mathrm{th}}\end{cases}
$$

where $P(m)$ denotes the outage probability of $m$ th MU$\mathrm{Rx}$, and $I_{\mathrm{ac}}$ denotes the actual interference from femtocells to macrocells (i.e., $I_{\mathrm{ac}}=\sum_{k} \sum_{i} p_{i}^{* k} G_{i}^{k}$, where $p_{i}^{* k}$ denotes the optimal transmit power of FBS). When $I_{\mathrm{ac}}<I_{\mathrm{th}}$, there is no outage; otherwise, the actual outage can be calculated by $\frac{I_{\text {ac }}-I_{\text {th }}}{I_{\text {th }}} \times 100 \%$.

To improve system capacity and spectrum efficiency, we formulate the following total transmit power minimization problem of FUs for uplink transmission model of two-tier HetNets, i.e.,

\section{Nominal optimization problem (P1)}

$$
\text { s.t. }\left\{\begin{array}{l}
\min _{p_{i}^{k} \in \Omega_{n}} \sum_{k=1}^{K} \sum_{i=1}^{M} p_{i}^{k} \\
C_{1}: \sum_{k=1}^{K} \sum_{i=1}^{M} p_{i}^{k} G_{i}^{k} \leq I_{\mathrm{th}}, \\
C_{2}: \gamma_{i}^{k} \geq \gamma_{i}^{k, \min }, \\
C_{3}: p_{i}^{k} \leq p_{i}^{k, \max } .
\end{array}\right.
$$

where $\Omega_{n}$ denotes the feasible region of P1 ( i.e., nonrobust optimization problem). To achieve these goals, we should discuss $\Omega_{n}$ when system information is exactly obtained. Obviously, when $I_{\text {th }}$ is extremely small, the feasible solution may not exist since FUs are very close to the MU-Rx. On the one hand, FUs cannot be allowed to transmit high power in order to guarantee MU's QoS. On the other hand, FUs need to improve their transmission power for their SINR requirement. Hence, we analyze the feasible case for satisfying the QoS of both FUs and MUs.

Remark 1 Let $\boldsymbol{p}^{l}=\left[p_{1}, \ldots, p_{L}\right]^{T}, \boldsymbol{p}^{k, \max }=\left[p_{1}^{k, \max }, \ldots\right.$, $\left.p_{M}^{k, \text { max }}\right]^{T}, \boldsymbol{m}=\left[\gamma_{1}^{k, \text { min }} \sigma_{1}^{k} / h_{1}^{k}, \ldots, \gamma_{M}^{k, \text { min }} \sigma_{M}^{k} / h_{M}^{k}\right]^{T}$ and $\boldsymbol{g}=\left[g_{i j}\right]=\left[\gamma_{i}^{k, \text { min }} g_{l}^{k} / h_{i}^{k}\right] . \boldsymbol{h}$ is the $M \times M$ intra-tier channel gain matrix with $\left[h_{i j}\right]=\left\{\begin{array}{ll}h_{j}^{k} / h_{i}^{k} & \text { if } j \neq i \\ 0 & \text { if } j=i\end{array} . \boldsymbol{F}\right.$ is a $M \times M$ gain matrix of FUs whose elements are $\boldsymbol{F}=\left[F_{i j}\right]=\left\{\begin{array}{ll}\gamma_{i}^{k, m i n} h_{i j} & \text { if } j \neq i, \\ 0 & \text { if } j=i .\end{array}\right.$. From constraint (3), we have $\boldsymbol{p}^{k, \text { min }}=(\boldsymbol{I}-\boldsymbol{F})^{-1}\left(\boldsymbol{g} \boldsymbol{p}^{l}+\boldsymbol{m}\right)$, where $\boldsymbol{p}^{k, \text { min }}=$ $\left[p_{1}^{k, \text { min }}, \ldots, p_{M}^{k, \text { min }}\right]^{T}$ denotes the minimum transmission power of FUs in the kth femtocell, and $I$ is a $M \times M$ unit matrix. The P1 is feasible if and only if the following conditions hold [18]:

$$
\Omega_{n}=\left\{\begin{array}{l}
\rho(\boldsymbol{F})<1, \\
\sum_{k}^{K} \boldsymbol{G}^{k} \boldsymbol{p}^{k} \leq I_{t h}, \\
p^{k, \text { min }} \leq \boldsymbol{p}^{k} \leq \boldsymbol{p}^{k, \max }
\end{array}\right.
$$

where $\boldsymbol{p}^{k}=\left[p_{1}^{k}, \ldots, p_{M}^{k}\right]^{T}$ is the feasible solution of $\boldsymbol{P} \mathbf{1}, \rho(\boldsymbol{F})$ is the spectral radius of $\boldsymbol{F}[19]$ and $\boldsymbol{G}^{k}=\left[G_{i}^{k}, \ldots, G_{M}^{k}\right]$ denotes channel gain vector between FU-Txs and MU-Rxs.

If channel gains in $C_{1}$ and $C_{2}$ can be perfectly known, P1 can be proved to be a convex optimization problem, which is easily solved under the feasible region $\Omega_{n}$ by the existing scheme, such as [20]. However, in practical dynamic communication environment, channel gains are actually uncertain that can influence system performance. For example, channel uncertainties between FU-Txs and MU-Rxs may bring the harmful interference to MUs, even 
cause in outage. Therefore, it is necessary to study RPA problem.

\section{Robust power allocation model}

In this section, the uncertainties of channel gains in P1 are considered and we use bounded ellipsoidal uncertainty sets to model them. Then, the SIP problem is transformed into a deterministic convex problem based on the CauchySchwartz inequality theory and worst-case approach.

\subsection{Models of channel uncertainties}

In practical systems, due to the effect of channel fading and feedback delays, the CSI is uncertain, which can be assumed to have a bounded uncertainty of unknown distribution. Ellipsoidal set is widely used to approximate unknown and potentially complicated uncertainty sets [21]. For example, for OFDM-based cognitive radio networks, the author in [22] proposed a worst-case robust distributed PA scheme, which employs the ellipsoidal approximate method to model the channel uncertainties. In [23], based on game theory, the author presented a robust optimization equilibrium for competitive rate maximization under bounded channel uncertainty and formulated the imperfect CSI by using ellipsoidal uncertainty sets. According to those existing literatures, it is obvious to see that the ellipsoidal approximation has the advantage of parametrically modeling complicated data sets and provides a convenient input parameter to algorithms. Furthermore, there are statistical reasons that lead to ellipsoidal uncertainty sets and also result in optimization problems with convenient analytical structures [24].

Therefore, by using ellipsoidal approximation, each uncertain parameter can be written as the sum of its nominal value and perturbation part, e.g.,

$$
h_{i j}=\bar{h}_{i j}+\Delta h_{i j},
$$

where $h_{i j}$ is the normalized intra-tier interference channel gain relevant to channel gain of link $i . \bar{h}_{i j}$ is the nominal value of channel gain between active FU-Rx and other FU-Txs from neighbor femtocells, and $\Delta h_{i j}$ is the corresponding perturbation part.

Let $H_{i}$ represent the uncertainty set of the $i$ th row of matrix $\mathbf{h}$. We use an ellipsoid set to describe $H_{i}$. Additionally, we denote $\overline{\mathbf{h}}=\left[\bar{h}_{i j}\right]$ and $\Delta \overline{\mathbf{h}}=\left[\Delta \bar{h}_{i j}\right]$. Under ellipsoid approximation, the uncertainty set of $H_{i}$ can be written as

$$
H_{i}=\left\{\overline{\mathbf{h}}_{i}+\Delta \mathbf{h}_{i}: \sum_{j \neq i}\left|\Delta h_{i j}\right|^{2} \leq \varepsilon_{i}^{2}\right\}
$$

where $\overline{\mathbf{h}}_{i}$ is the $i$ th row of $\overline{\mathbf{h}}$, and the corresponding perturbation part as $\Delta \mathbf{h}_{i}$, and $\varepsilon_{i} \geq 0$ is the maximum evaluated error of every row in $\overline{\mathbf{h}}_{i}$.
Similarly, the uncertainty relevant to the interference channel gain between FU and MU-Rx can be written as

$$
G_{i}^{k}=\bar{G}_{i}^{k}+\Delta G_{i}^{k} \text {, }
$$

where $\bar{G}_{i}^{k}$ and $\Delta G_{i}^{k}$ represent the nominal value and the perturbation part of channel gain between $\mathrm{FU}$ and MU$\mathrm{Rx}$, respectively.

Let $G_{i}$ represent the uncertainty set of the $i$ th column of matrix $\mathbf{G}=\left[G_{1}^{1} \cdots G_{M}^{1} ; \cdots ; G_{1}^{K} \cdots G_{M}^{K}\right]$. Denote the $i$ th column of $\overline{\mathbf{G}}$ and the corresponding perturbation part as $\overline{\mathbf{G}}_{i}$ and $\Delta \mathbf{G}_{i}$, respectively. The uncertainty parameter $G_{i}$ is described by an ellipsoid set as follows

$$
G_{i}=\left\{\overline{\mathbf{G}}_{i}+\Delta \mathbf{G}_{i}: \sum_{k}\left|\Delta G_{i}^{k}\right|^{2} \leq \delta_{i}^{2}\right\} .
$$

where $\delta_{i} \geq 0$ is the maximum deviation of each item in $\overline{\mathbf{G}}_{i}$.

Furthermore, we also consider uncertainties of the normalized cross-tier interference channel gains from MU-Tx to FU-Rx.

$$
g_{i l}=\bar{g}_{i l}+\Delta g_{i l}
$$

where $\bar{g}_{i l}$ is the nominal value, and $\Delta g_{i l}$ is the perturbation part. Let $g_{i}$ represent the uncertainty sets of the $i$ th row of matrix $\mathbf{g}$. Denote the $i$ th row of $\overline{\mathbf{g}}$ as $\overline{\mathbf{g}}_{i}$, and the corresponding perturbation part as $\Delta \mathbf{g}_{i}$. In this case, the uncertainty region is given as

$$
g_{i}=\left\{\overline{\mathbf{g}}_{i}+\Delta \mathbf{g}_{i}: \sum_{l}\left|\Delta g_{i l}\right|^{2} \leq \omega_{i}^{2}\right\} .
$$

where $\omega_{i} \geq 0$ is the maximum deviation of each row in $\overline{\mathbf{g}}_{i}$.

\subsection{Robust power allocation optimization model}

Considering the channel uncertainties, the RPA problem is formulated as

Robust power allocation problem (P2)

$$
\begin{aligned}
& \min _{p_{i}^{k} \in \Omega_{r}} \sum_{k=1}^{K} \sum_{i=1}^{M} p_{i}^{k} \\
& \left\{\begin{array}{l}
C_{4}: \sum_{k=1}^{K} \sum_{i=1}^{M}\left(\bar{G}_{i}^{k}+\Delta G_{i}^{k}\right) p_{i}^{k} \leq I_{t h}, \\
C_{5}: \frac{\sum_{j \neq i}^{M}\left(\bar{h}_{i j}+\Delta h_{i j}\right) p_{j}^{k}+\sum_{l=1}^{L}\left(\bar{g}_{i l}+\Delta g_{i l}\right) p_{l}+\frac{\sigma_{i}^{k}}{h_{i}^{k}}}{p_{i}^{k}} \leq \frac{1}{\gamma_{i}^{k, \min },} \\
C_{6}: p_{i}^{k} \leq p_{i}^{k, \max }, \\
C_{7}: \sum_{j \neq i}\left|\Delta h_{i j}\right|^{2} \leq \varepsilon_{i}^{2}, \\
\sum_{i}\left|\Delta G_{i}\right|^{2} \leq \delta_{i}^{2}, \\
\sum_{l}\left|\Delta g_{i l}\right|^{2} \leq \omega_{i}^{2} .
\end{array}\right.
\end{aligned}
$$

where $\Omega_{r}$ denotes the feasible region of RPA problem. Since $\mathbf{P 2}$ is limited by an infinite number of constraints like sets $H_{i}, G_{i}$, and $g_{i}, \mathbf{P} 2$ is proved to be a SIP problem [10]. A feasible method to solve the SIP problem is to transform it into a deterministic robust problem by considering the worst case in the constraints of P2. In other 
words, we can keep the system performance under any case of estimation errors.

According to the Cauchy-Schwartz inequality theory and worst-case approach [25], the uncertain part of $C_{4}$ and $C_{5}$ can be converted into

$$
\max \left\{\sum_{k=1}^{K} \sum_{i=1}^{M} \Delta G_{i}^{k} p_{i}^{k}\right\} \leq \delta_{i} \sqrt{\sum_{k=1}^{K} \sum_{i=1}^{M}\left(p_{i}^{k}\right)^{2}} \leq \delta_{i} \sum_{k=1}^{K} \sum_{i=1}^{M} p_{i}^{k},
$$

$$
\left\{\begin{array}{l}
\max \left\{\sum_{j \neq i}^{M} \Delta h_{i j} p_{j}^{k}\right\} \leq \varepsilon_{i} \sqrt{\sum_{j \neq i}^{M}\left(p_{j}^{k}\right)^{2}}, \\
\max \left\{\sum_{l=1}^{L} \Delta g_{i l} p_{l}\right\} \leq \omega_{i} \sqrt{\sum_{l=1}^{L} p_{l}^{2} .}
\end{array}\right.
$$

Based on (15) and (18), the RPA problem (P2) can be reformulated as follows

Worst-case power allocation problem (P3)

$$
\begin{array}{ll} 
& \min _{p_{i}^{k} \in \Omega_{r}} \sum_{k=1}^{K} \sum_{i=1}^{M} p_{i}^{k} \\
\boldsymbol{C}_{7}: I_{N} \leq 1, \\
C_{8}: \frac{Z_{i}^{k}+E_{i}^{k}}{p_{i}^{k}} \leq \frac{1}{\gamma_{i}^{k, \min }} \\
C_{9}: p_{i}^{k} \leq p_{i}^{k, \max } .
\end{array}
$$

where

$$
\begin{aligned}
I_{N} & =\sum_{k=1}^{K} \sum_{i=1}^{M}\left(\bar{G}_{i}^{k}+\delta_{i}\right) p_{i}^{k} / I_{t h .} \\
E_{i}^{k} & =\varepsilon_{i} \sqrt{\sum_{j \neq i}^{M}\left(p_{j}^{k}\right)^{2}}+\omega_{i} \sqrt{\sum_{l=1}^{L} p_{l^{2}} .} \\
Z_{i}^{k} & =\sum_{j \neq i}^{M} \bar{h}_{i j} p_{j}^{k}+\sum_{l=1}^{L} \bar{g}_{i l} p_{l}+\frac{\sigma_{i}^{k}}{h_{i}^{k}} .
\end{aligned}
$$

It is obvious that the above $\mathbf{P 3}$ is a convex problem with liner constraints. To get an insight on the solution to P3 and compare it with that of the nominal problem (i.e., P1), we need now study the feasibility region of the robust problem. According to the feasible region of non-robust problem [i.e., (7)], we derive the robust feasible region with the following form:

$$
\Omega_{r}=\left\{\begin{array}{l}
\rho(\overline{\mathbf{F}})+\left\|\boldsymbol{\Delta}_{j i}\right\|_{F}<1, \quad(21 a) \\
\sum_{k}^{K} \overline{\mathbf{G}}^{k} \tilde{\mathbf{p}}^{k^{T}}+\$[\tilde{\mathbf{p}} \delta] \leq I_{\mathrm{th}}, \\
\mathbf{p}^{k, \text { min }} \leq \tilde{\mathbf{p}}^{k} \leq \mathbf{p}^{k, \max }
\end{array}\right.
$$

where $\$[\bullet]$ denotes the sum of matrix elements, $\overline{\mathbf{F}}$ denotes the nominal matrix of Remark 1, $\tilde{\mathbf{p}}=\left[p_{i}^{k}\right]=$ $\left[p_{1}^{1}, \ldots, p_{M}^{1} ; \ldots ; p_{1}^{K}, \ldots, p_{M}^{K}\right]$ is the feasible solution of RPA problem (i.e., P3) and $\tilde{\mathbf{p}}^{k}$ is the $k$ th row of matrix $\tilde{\mathbf{p}}$ $. \overline{\mathbf{G}}^{k}=\left[\bar{G}_{1}^{k}, \ldots, \bar{G}_{M}^{k}\right]$ and $\boldsymbol{\delta}=\left[\delta_{1}, \ldots, \delta_{M}\right]^{T}$ denote the nominal cross-tier channel estimates and maximum channel perturbation, respectively. Obviously, conditions (21c) are satisfied. The proof of (21a) and (21b) is given in Appendix A.

\section{Robust power allocation algorithm}

In this section, we will propose a RPA algorithm to solve P3 by applying the decomposition theory. The Lagrange function of P3 is defined as

$$
\begin{aligned}
L\left(\left\{p_{i}^{k}\right\}, \lambda,\left\{\mu_{i}^{k}\right\},\left\{\xi_{i}^{k}\right\}\right)= & \sum_{k=1}^{K} \sum_{i=1}^{M} p_{i}^{k}+\lambda\left(I_{N}-1\right) \\
& +\sum_{k=1}^{K} \sum_{i=1}^{M} \mu_{i}^{k}\left(\frac{Z_{i}^{k}+E_{i}^{k}}{p_{i}^{k}}-\frac{1}{\gamma_{i}^{k, \min }}\right) \\
& +\sum_{k=1}^{K} \sum_{i=1}^{M} \xi_{i}^{k}\left(p_{i}^{k}-p_{i}^{k, \max }\right) .
\end{aligned}
$$

where $\lambda,\left\{\mu_{i}^{k}\right\}$ and $\left\{\xi_{i}^{k}\right\}$ are Lagrange multipliers and $\lambda \geq$ $0, \mu_{i}^{k} \geq 0, \xi_{i}^{k} \geq 0$. And the dual Lagrange function is

$$
\begin{aligned}
D\left(\left\{p_{i}^{k}\right\}, \lambda,\left\{\mu_{i}^{k}\right\},\left\{\xi_{i}^{k}\right\}\right)= & \min _{p_{i}^{k}} L\left(\left\{p_{i}^{k}\right\}, \lambda,\left\{\mu_{i}^{k}\right\},\left\{\xi_{i}^{k}\right\}\right) \\
= & \sum_{k} \sum_{i} \min L_{i}^{k}\left(p_{i}^{k}, \lambda, \mu_{i}^{k}, \xi_{i}^{k}\right) \\
& -\lambda-\sum_{i} \sum_{k} \mu_{i}^{k} \frac{1}{\gamma_{i}^{k, \min }} \\
& -\sum_{i} \sum_{k} \xi_{i}^{k} p_{i}^{k, \max },
\end{aligned}
$$

where

$$
L_{i}^{k}\left(p_{i}^{k}, \lambda, \mu_{i}^{k}, \xi_{i}^{k}\right)=p_{i}^{k}+\lambda I_{N}+\mu_{i}^{k}\left(\frac{Z_{i}^{k}+E_{i}^{k}}{p_{i}^{k}}\right)+\xi_{i}^{k} p_{i}^{k} .
$$

and the dual optimization problem is formulated as

$$
\begin{aligned}
& \max _{\lambda, \mu_{i}^{k}, \xi_{i}^{k}} D\left(\lambda,\left\{\mu_{i}^{k}\right\},\left\{\xi_{i}^{k}\right\}\right) \\
& \text { s.t. } \lambda \geq 0, \mu_{i}^{k} \geq 0, \xi_{i}^{k} \geq 0 .
\end{aligned}
$$

For any FUs, the dual decomposition method can be separated into some sub-problems with parallel form. Since $L_{i}^{k}\left(p_{i}^{k}, \lambda, \mu_{i}^{k}, \xi_{i}^{k}\right)$ is a convex problem with respect to $p_{i}^{k}$. According to the KKT condition [25], the optimal 
transmit power $p_{i}^{k *}$ can be calculated by $\frac{\partial L_{i}^{k}\left(p_{i}^{k}, \lambda, \mu_{i}^{k}, \xi_{i}^{k}\right)}{\partial p_{i}^{k}}=0$ and the result is

$$
p_{i}^{k *}=\sqrt{\frac{\mu_{i}^{k}\left(Z_{i}^{k}+E_{i}^{k}\right)}{1+\xi_{i}^{k}+\lambda \sum_{k=1}^{K} \sum_{i=1}^{M}\left(\bar{G}_{i}^{k}+\delta_{i}\right) / I_{\mathrm{th}}}} .
$$

Define

$$
\begin{aligned}
& S_{\lambda}=\sum_{k=1}^{K} \sum_{i=1}^{M}\left(\bar{G}_{i}^{k}+\delta_{i}\right) p_{i}^{k *} / I_{\mathrm{th}} \\
& S_{\mu_{i}^{k}}=\frac{Z_{i}^{k}+E_{i}^{k}}{p_{i}^{k *}}-\frac{1}{\gamma_{i}^{k, \min }} \\
& S_{\xi_{i}^{k}}=p_{i}^{k *}-p_{i}^{k, \max }
\end{aligned}
$$

where $S_{\lambda}, S_{\mu_{i}^{k}}$, and $S_{\xi_{i}^{k}}$ are the sub-gradients of $\lambda, \mu_{i}^{k}$, and $\xi_{i}^{k}$, respectively.

Update $p_{i}^{k *}(t+1)$ and Lagrange multipliers $\lambda, \mu_{i}^{k}$, and $\xi_{i}^{k}$ as follows

$$
\begin{aligned}
& p_{i}^{k *}(t+1)=\min \left\{p_{i}^{k, \max }, p_{i}^{k *}(t)\right\} . \\
& \lambda(t+1)=\left[\lambda(t)+\alpha S_{\lambda}\right]^{+} \\
& \mu_{i}^{k}(t+1)=\left[\mu_{i}^{k}(t)+\beta S_{\mu_{i}^{k}}\right]^{+} \\
& \xi_{i}^{k}(t+1)=\left[\xi_{i}^{k}(t)+\theta S_{\xi_{i}^{k}}\right]^{+}
\end{aligned}
$$

where $[x]^{+}=\max \{0, x\}, \alpha, \beta$, and $\theta$ are the step sizes which are positive and $t$ is the step time (Table 2). The outline of our proposed RPA algorithm is described in the Table 2.

\section{Performance analysis}

\subsection{Computational complexity}

For the specific variable $(i, k)$, the convergence times of finding the optimal solution $p_{i}^{k *}$ via Newton iterative approach is assumed to be $t_{1}$ for sub-problem. As the dual problems can be decomposed into $M \times N$ subproblems, the sum iteration number of total sub-problems is $M \times N \times t_{1}$, for all $(i, k)$. In addition, from (31)-(33), we need the $(2 M N+1)$ steps to update the Lagrange multipliers. The iteration number of finding the optimal
Table 2 Proposed RPA algorithm

Proposed algorithm

$1:$ Initialize maximum iteration number $T_{\text {max }}$; Set: iteration $t=0, M>0$, and $L>0$; Lagrangian

multipliers $\lambda(0)>0,\left\{\mu_{i}^{k}\right\}(0)>0$, and $\left\{\xi_{i}^{k}\right\}(0)>0$; step sizes $\alpha>$ $0, \beta>0$, and $\theta>0$;

upper bound of estimation error in MU-to-FBS link is $\omega_{i} \in[0,0.003]$; upper bound of estimation error in femtocell link is $\epsilon_{i} \in[0,0.1]$; upper bound of estimation error in FU-to-MBS link is $\delta_{i} \in[0,0.003]$.

2:Set maximum transmit power $p_{i}^{k, \max }>0$ and initialize power $p_{i}^{k}>0$ with different initialization

values among different FUs and MUs.

3:Define interference $I_{\text {th }}$ and minimum rate $\gamma_{i}^{k, m i n}$, randomly generate $\bar{G}_{i}^{k}, \bar{h}_{i j}$ and $\bar{g}_{i j}$.

4.repeat

5: for $t=1$ to $T_{\max }$ do

6: for $m=1$ to $M$ do

7: $\quad$ for $/=1$ to $L$ do

8: $\quad$ Calculate transmit power $p_{i}^{k *}$ according to (26):

9: $\quad$ Calculate actual SINR received at FU-Rx according to (2) and (26);

10: Calculate $l_{a c}$ according to (1) and (26);

11: Calculate Lagrange multipliers $\lambda, \mu_{i}^{k}$, and $\xi_{i}^{k}$ from (31) - (33);

12: end for

13: end for

$3 t=t+1$.

14:end for

15:until $t=T_{\text {max }}$ or transmit power convergence.

variables $\left(\lambda^{*}, \mu_{i}^{k *}, \xi_{i}^{k *}\right)$ is assumed to be $t_{2}$. Hence, the complexity of our proposed algorithm can be expressed as $\mathcal{O}\left(\left(M N t_{1}+2 M N+1\right) t_{2}\right)$.

\subsection{Sensitivity analysis}

In this sub-section, we use local sensitivity analysis of P3 by perturbing its constraints. For all value of $\Delta h_{i}^{j}, \Delta G_{i}^{k}$, and $\Delta g_{i}^{l}$, the reduction of achievable sum transmit power can be approximated as

$$
P_{\Delta} \approx-\sum_{i=1}^{M} \lambda^{*} \delta_{i}-\sum_{i=1}^{M} \sum_{k=1}^{N}\left(\mu_{i}^{k *} \varepsilon_{i}+\mu_{i}^{k *} \omega_{i}\right) .
$$

where $\lambda^{*}$ and $\mu_{i}^{k *}$ denote optimal Lagrange multipliers. The proof is given in Appendix B.

\section{Numerical results}

In this section, the simulation results and performance analysis are provided to verify the efficiency and performance of our proposed algorithm. In this part, we used 
MATLAB 2016 software to do the simulations via core i5. In our simulation, we assumed that actual channel fading follows Rayleigh fading model; therefore, actual channel gains $G_{i}^{k}, h_{i}^{k}$, and $g_{i}^{k}$ are followed as $\left\{0, \frac{A}{d^{r}}\right\}$, where $d$ is the distance from transmitter to receiver, $r \in$ $[2,5]$ denotes the path-loss exponent, and the attenuation parameter $A$ is frequency dependent [26]. The traditional non-robust PA algorithm is given in [5] under perfect CSI. Due to not taking into account the channel uncertainty, our RPA algorithm has more advantages in improving network performance compared with the non-robust PA algorithm. Other simulation parameters are given in Table 3.

Figure 2 presents the transmission power of each FU under multiuser scenarios, such as $M=3$, and channel uncertainties are $\varepsilon_{i}$, and $\omega_{i}$ are supposed to be $1 \times 10^{-3}$, and $\delta_{i}$ is assumed to be $10 \%$ of $\bar{G}_{i}^{k}$. As can be seen in Fig. 2, with the increasing iteration numbers, the transmit power increases and tends to be converging to a stable value when the iteration number is about six, which demonstrates the perfect convergence performance of our proposed algorithm. In addition, transmit power is restricted by the maximum value $p_{i}^{k \text {,max }}$, which shows the proposed algorithm is feasible. As a result, it satisfies the maximum power constraint (4).

To demonstrate the effectiveness of our proposed algorithm in term of QoS protection of both FUs and MUs, we also give the comparison of performance between the proposed RPA algorithm and traditional non-robust PA algorithm [5].

Figure 3 shows comparison between our RPA algorithm and non-robust PA algorithm in terms of SINR. It is obvious that the SINR of each FU under our proposed algorithm exceeds the minimum SINR value with considering the estimation errors, whereas the non-robust PA algorithm cannot guarantee SINR requirements of all FUs, which will lead to a communication outage. Due to the effects of channel fading and feedback delays, FUs cannot respond in time by using traditional PA algorithm

Table 3 Simulation parameters

\begin{tabular}{ll}
\hline System parameter & Values \\
\hline Number of MUEs $L$ & 4 \\
Number of FBSs $M$ & 1 \\
Number of FUEs in each femtocell $M$ & 3 \\
The background noise $\sigma_{i}^{k}$ & $10^{-8} \mathrm{~W}$ \\
Minimum SINR rquirement of FUs $\gamma_{i}^{k, \text { min }}$ & $2 \mathrm{~dB}$ \\
Transmit power of each MU $p_{l}$ & {$[0.5,1] \mathrm{W}$} \\
Maximum transmit power $p_{i}^{k, \max }$ & $1 \mathrm{~W}$ \\
Allowable interference level $I_{\text {th }}$ & $10^{-3} \mathrm{~W}[25]$ \\
\hline
\end{tabular}

so that the QoS of each FU is hard to guarantee. Moreover, it indicates that RPA algorithm can always ensure the normal communication of FUs. Therefore, the robustness of our proposed RPA algorithm is better than the traditional PA algorithm without consideration of channel uncertainties.

Figure 4 gives comparison of the interference power received at MBS between our proposed robust algorithm and the non-robust algorithm. As shown in Fig. 4, the interference power introduced to the MBS with considering channel uncertainties is always under the interference power threshold, whereas the actual received interference power at MBS under non-robust PA algorithm exceeds the tolerable region. It can be explained that the MUs may experience severe performance degradation. Therefore, QoS of MUs are not guaranteed without the consideration of estimation errors and an outage event happens.

Figure 5 provides comparison of total energy consumption under our proposed RPA algorithm and traditional non-robust PA algorithm. From Fig. 5, the total power consumption of FUs in both non-robust algorithm and RPA algorithm increase with the increasing number of iteration and converge to a stable value; however, the total transmit power of RPA algorithm is higher than that of non-robust PA algorithm. From Figs. 3, 4, and 5 , we can get a conclusion that our RPA algorithm can well protect the QoS of MUs at the expense of energy consumption.

Considering imperfection of actual CSI, in order to demonstrate the superiority of our proposed RPA algorithm under different channel uncertainty (i.e., $\delta_{i}, \varepsilon_{i}, \omega_{i}$ ) clearly, we give the satisfaction probability of MU-Rx and SINR performance of FUs in Figs. 6, 7, and 8.

Figure 6 shows that the satisfaction probability of MURx using two different algorithms can be presented subject to different channel estimation errors $\Delta G_{i}^{k}$. It is clear that the satisfaction probability of MU-Rx under the non-robust PA algorithm is rapidly declining as the increasing interference channel uncertainty $\delta_{i}$. Whereas the system with our RPA algorithm can cope with this problem. This is because the RPA algorithm is adaptive that can adjust $p_{i}^{k}$ according to channel perturbation $\delta_{i}$. In addition, bigger interference threshold $I_{\text {th }}$ of MU-Rx can increase the feasible region of transmit power $p_{i}^{k}$ and then decrease the outage probability of MU-Rx. While satisfaction probability of MU using non-robust PA algorithm is lower than that of MU using our RPA algorithm, in other words, non-robust PA algorithm can increase the outage probability of MUs. Hence, it enables the proposed RPA algorithm to protect the normal communication of MUs.

Figure 7 shows the actual SINR received at FU-Rx under inter-tier and cross-tier channel uncertainties (i.e., $\varepsilon_{i}$ and 


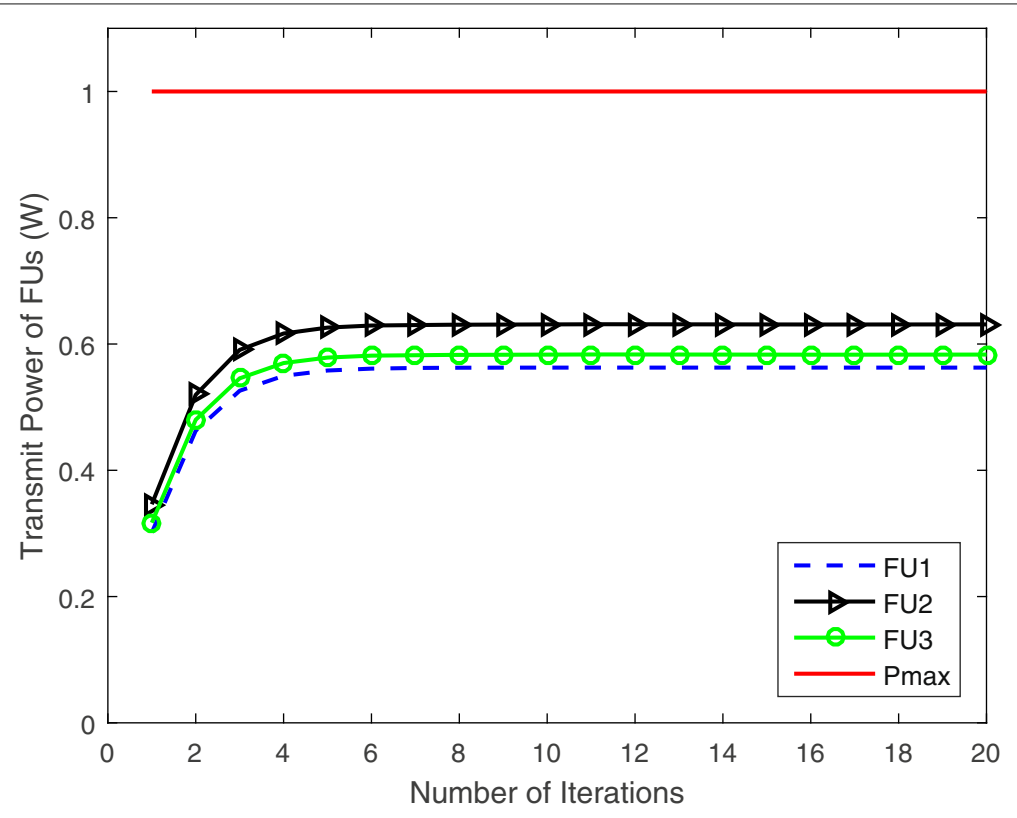

Fig. 2 Transmit power versus number of iterations

$\left.\omega_{i}\right)$ by two algorithms. The bound of the interference perturbation is $\delta_{i}=0.0001$. As can be seen from Fig. 7, the received SINR of FU-Rx decreases with the increase of inter-tier channel perturbation $\varepsilon_{i}$ and cross-tier channel perturbation $\omega_{i}$ by our RPA algorithm and non-robust PA algorithm. The reason is that optimal power reduction leads to the decrease of received SINR. Additionally, it is obvious that the received SINR in [5] is lower than the received SINR by using our proposed algorithm. And when $\varepsilon_{i}>0.045$, the received SINR in [5] cannot meet the minimum SINR requirement. What is more, received SINR of FU-Rx declines with cross-tier channel uncertainties $\omega_{i}$ increase. That is due to traditional non-robust PA algorithm ignores channel estimation errors, and $p_{i}^{k}$

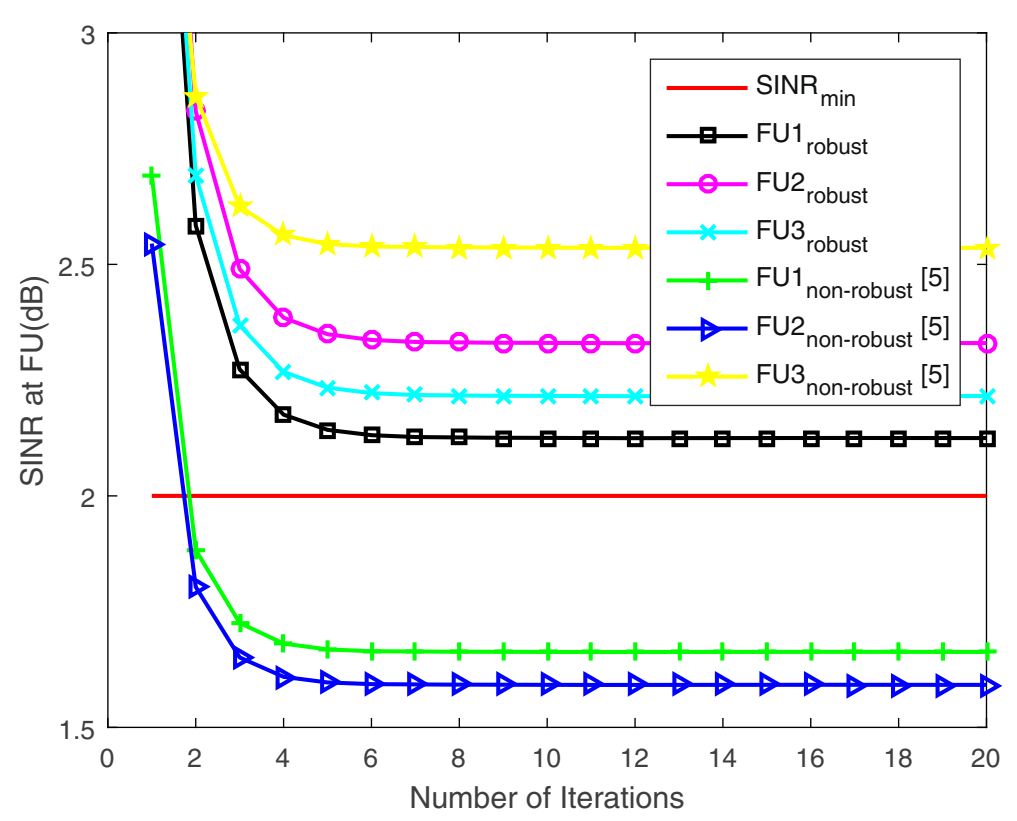

Fig. 3 Comparison of SINR received at FBS between robust and non-robust algorithm 


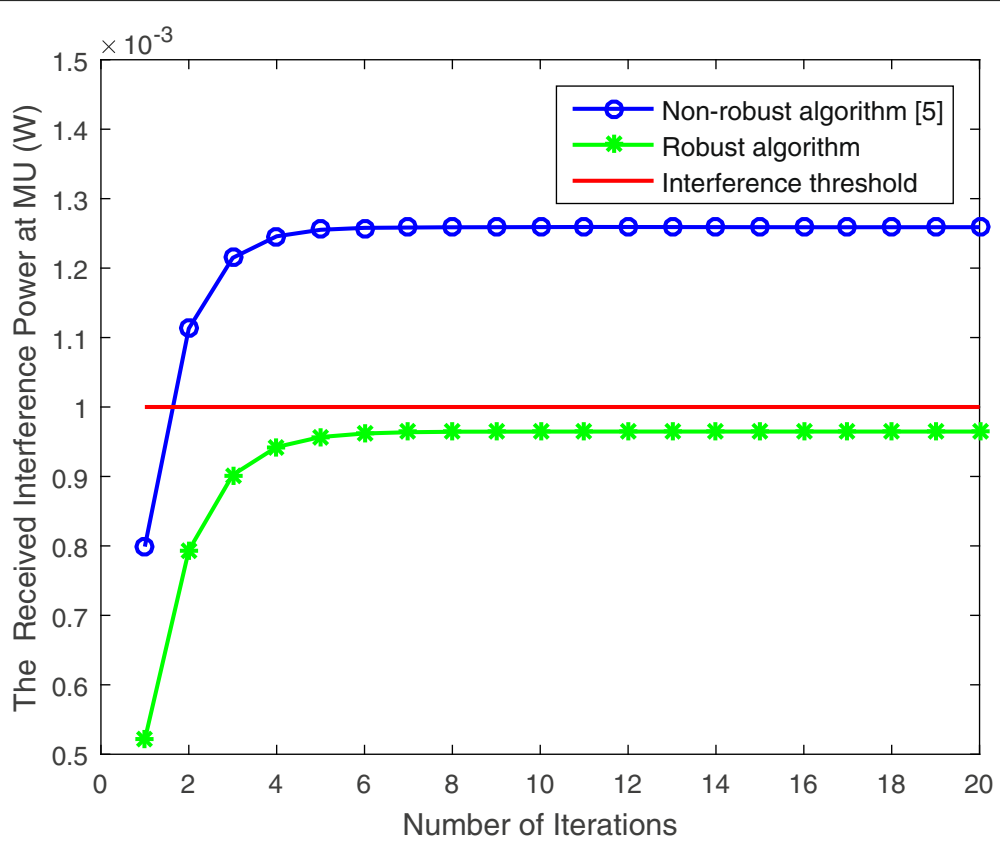

Fig. 4 Comparison of the interference power received at MU-Rx between robust and non-robust algorithm

cannot be adjusted in time under time-varying channel uncertainty.

Figure 8 presents the comparison of received SINR performance of FU-Rx between our proposed RPA algorithm and non-robust PA algorithm under channel uncertainties. From Fig. 8, we can see intuitively that the SINR performance of our algorithm is superior to that of non-robust algorithm and cannot lead to the interruption of communication when channel environment is bad. In conclusion, the proposed RPA algorithm can improve

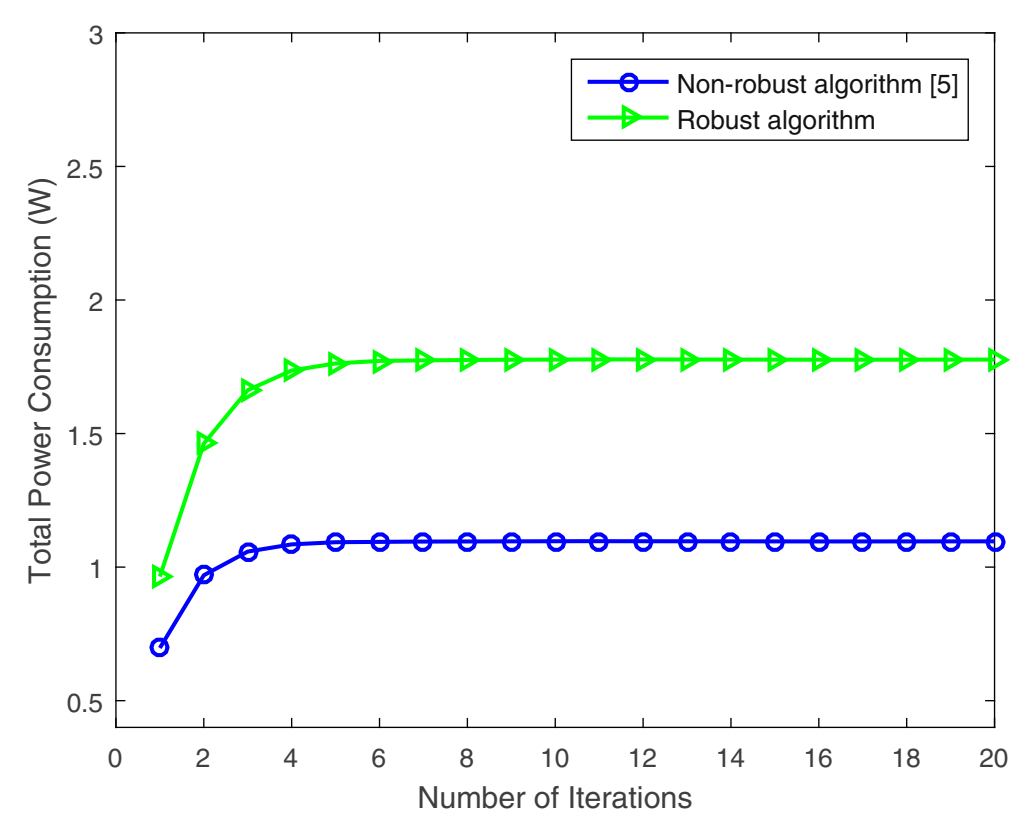

Fig. 5 Comparison of total power consumption between robust and non-robust PA method 


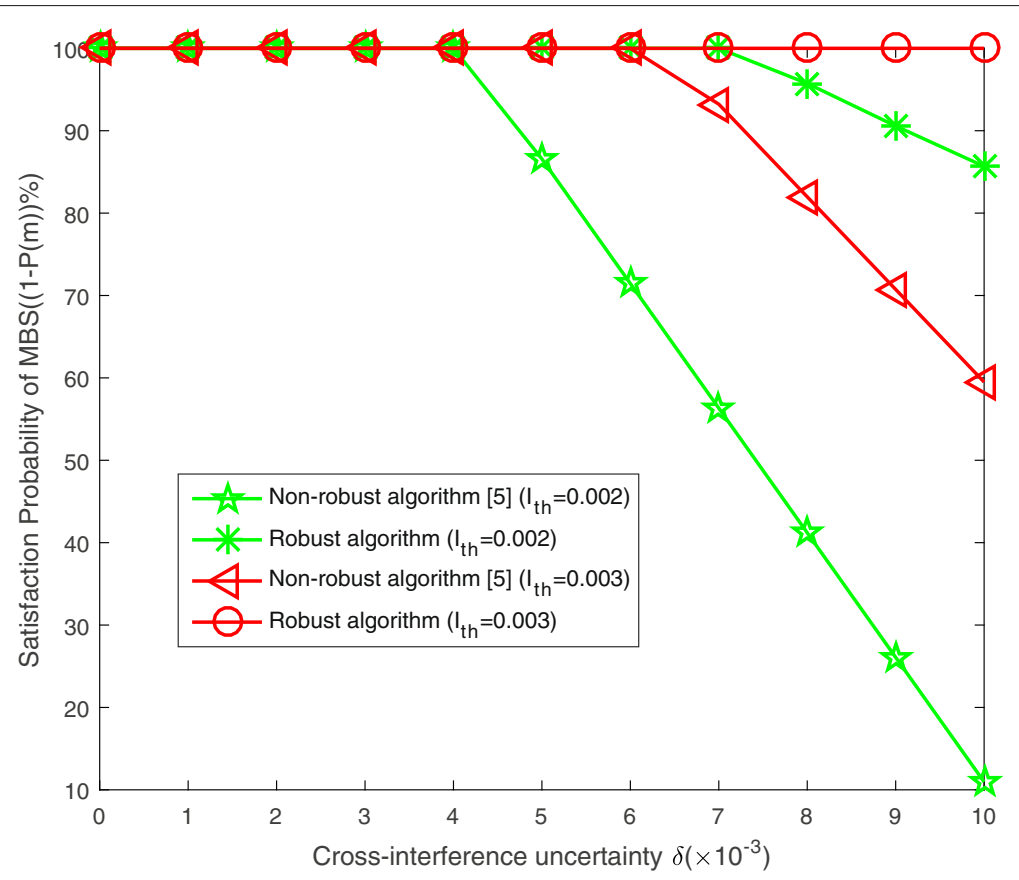

Fig. 6 Outage probability at MU-Rx between robust and non-robust algorithm under different interference perturbation $\delta_{i}$

the quality of communication system compared with the traditional non-robust PA algorithm under channel uncertainties.

\section{Conclusions}

In this paper, a RPA problem is studied in uplink two-tier HetNets with all possible channel uncertainties. Based on the worst-case approach, the robust resource optimization problem is converted into a convex one which is solved by using Lagrange dual method. The feasible regions and the closed analytical solution are obtained. Furthermore, performance analysis and the impact of channel uncertainties have been presented. The numerical results show that our proposed RPA algorithm is out-performance to traditional non-robust algorithm in cases of protecting the QoS of MUs at the cost of energy loss. In our future work, we will extend the network structure for multiple macrocells and relay-assisted transmission cases.

\section{Appendix A}

Proof of condition (21a) According to the discussion of Remark 1, the minimum transmission power of $\boldsymbol{P 3}$ is

$$
\mathbf{p}^{k, \min }=\left(\mathbf{I}-\overline{\mathbf{F}}-\boldsymbol{\Delta}_{F}\right)^{-1}\left(\overline{\boldsymbol{g}} \boldsymbol{p}^{l}+\boldsymbol{\Gamma}+\mathbf{m}\right)
$$

where $\boldsymbol{\Gamma}=\left[\gamma_{1}^{k, \min } \omega_{1} \Psi, \ldots, \gamma_{M}^{k, \min } \omega_{M} \Psi\right]^{T}$ denotes the perturbation part of interference, and $\Psi=\sqrt{\sum_{l} p_{l}^{2}}$.
Additionally, $\boldsymbol{\Delta}_{F}$ is a $M \times M$ matrix whose elements

$$
\boldsymbol{\Delta}_{F}=\left[\Delta_{i j}\right]= \begin{cases}\gamma_{i}^{k, \min } \Delta h_{i j} & \text { if } j \neq i, \\ 0 & \text { if } j=i .\end{cases}
$$

According to $\mathbf{F}=\overline{\mathbf{F}}+\boldsymbol{\Delta}_{F}$ and $\rho(\mathbf{F})<1$, we have

$$
\rho\left(\overline{\mathbf{F}}+\boldsymbol{\Delta}_{F}\right)<1
$$

According to the definition of spectral radius and the property of Frobenius norm [27], we have

$$
\begin{aligned}
\left\|\Delta_{i j}\right\|_{F} & =\sqrt{\sum_{i} \sum_{j} \Delta_{i j}^{2}}, \\
\left\|\Delta_{i j}\right\|_{2} & \leq\left\|\Delta_{i j}\right\|_{F} .
\end{aligned}
$$

Combining with the triangle inequality [28], we have

$$
\begin{aligned}
\rho\left(\overline{\mathbf{F}}+\Delta_{F}\right) & =\left\|\bar{F}_{j i}+\Delta_{i j}\right\|_{2} \leq\left\|\bar{F}_{j i}\right\|_{2}+\left\|\Delta_{i j}\right\|_{2} \\
& \leq \rho(\overline{\mathbf{F}})+\left\|\Delta_{i j}\right\|_{F}<1 .
\end{aligned}
$$

Proof of condition (21b) Considering cross-tier channel uncertainties between FUs and MU-Rxs, the interference constraint condition of $\Omega_{r}$ is 


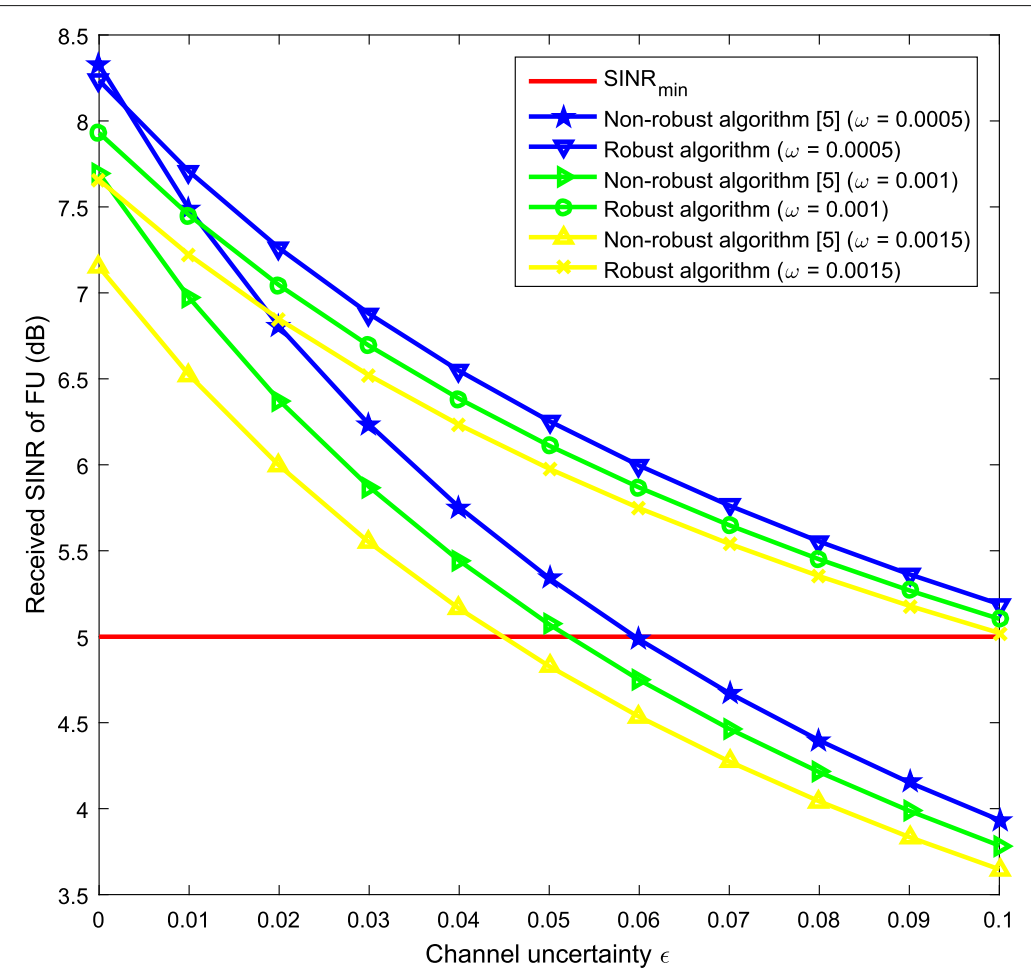

Fig. 7 Comparison of SINR between robust and non-robust algorithm under different channel perturbation $\epsilon$ and $\omega$

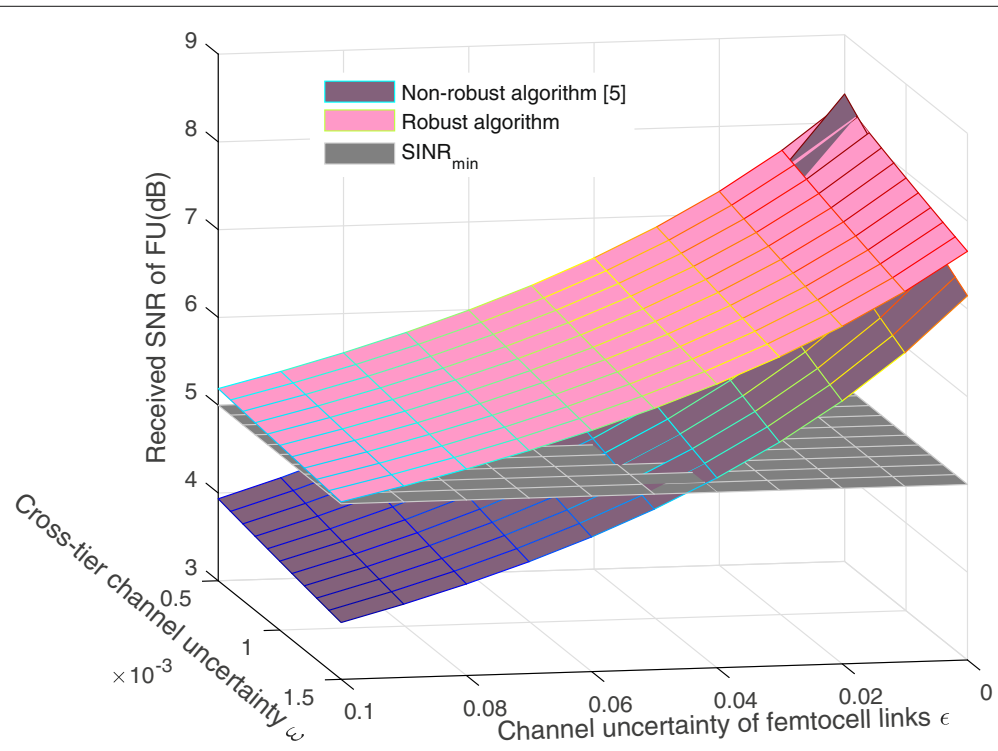

Fig. 8 SINR comparison of robust and non-robust algorithm under time-varying channel uncertainty 


$$
\sum_{k}^{K}\left(\overline{\mathbf{G}}^{k}+\Delta \mathbf{G}^{k}\right) \tilde{\mathbf{p}}^{k} \leq I_{\mathrm{th}} .
$$

According to inequality (11) and (15), we have

$$
\begin{aligned}
\sum_{k}^{K} \overline{\mathbf{G}}^{k} \tilde{\mathbf{p}}^{k}+\sum_{k}^{K} \Delta \mathbf{G}^{k} \tilde{\mathbf{p}}^{k} & \leq \sum_{k}^{K} \overline{\mathbf{G}}^{k} \tilde{\mathbf{p}}^{k}+\sum_{i}^{M} \delta_{i} \sum_{k}^{K} p_{i}^{k} \\
& =\sum_{k}^{K} \overline{\mathbf{G}}^{k} \tilde{\mathbf{p}}^{\mathrm{T}}+\mathbf{s}[\tilde{\mathbf{p}} \boldsymbol{\delta}] \leq I_{\mathrm{th}}
\end{aligned}
$$

With that, the proof of condition (21a) and (21b) are completed.

\section{Appendix B}

Based on the formula of Taylor series of the three element function, we have

$$
\begin{aligned}
& P^{*}\left(\bar{G}_{i}^{k}+\Delta G_{i}^{k}, \bar{h}_{i}^{k}+\Delta h_{i}^{j}, \bar{g}_{i}^{k}+\Delta g_{i}^{k}\right)=P^{*}\left(\bar{G}_{i}^{k}, \bar{h}_{i}^{k}, \bar{g}_{i}^{k}\right) \\
& +\sum_{i=1}^{M} \sum_{k=1}^{N} \frac{\partial P^{*}\left(\bar{G}_{i}^{k}, \bar{h}_{i}^{k}+\Delta h_{i}^{j}, \bar{g}_{i}^{k}+\Delta g_{i}^{k}\right)}{\partial \Delta G_{i}^{k}} \\
& +\sum_{i=1}^{M} \sum_{k=1}^{N} \frac{\partial P^{*}\left(\bar{G}_{i}^{k}+\Delta G_{i}^{k}, \bar{h}_{i}^{k}, \bar{g}_{i}^{k}+\Delta g_{i}^{k}\right)}{\partial \Delta h_{i}^{j}} \\
& +\sum_{i=1}^{M} \sum_{k=1}^{N} \frac{\partial P^{*}\left(\bar{G}_{i}^{k}+\Delta G_{i}^{k}, \bar{h}_{i}^{k}+\Delta h_{i}^{j}, \bar{g}_{i}^{k}\right)}{\partial \Delta g_{i}^{k}} \\
& +o,\left(\Delta G_{i}^{k} \rightarrow 0, \Delta h_{i}^{j} \rightarrow 0, \Delta g_{i}^{k} \rightarrow 0\right)
\end{aligned}
$$

where $o$ denotes the corresponding high order infinitesimal small quantities. And, $P^{*}\left(\bar{G}_{i}^{k}, \bar{h}_{i}^{k}, \bar{g}_{i}^{k}\right)$ is the optimal value for P3 without estimation errors (assuming that the estimated channel gains are equal to the actual channel gains).

Ignoring the effect of high order small variables, since P3 is convex, $P^{*}\left(\bar{G}_{i}^{k}+\Delta G_{i}^{k}, \bar{h}_{i}^{k}+\Delta h_{i}^{j}, \bar{g}_{i}^{k}+\Delta g_{i}^{k}\right)$ is obtained from the Lagrange dual function and using the sensitivity analysis [29], we have

$$
\begin{gathered}
\sum_{k=1}^{N} \frac{\partial P^{*}\left(\bar{G}_{i}^{k}, \bar{h}_{i}^{k}+\Delta h_{i}^{j}, \bar{g}_{i}^{k}+\Delta g_{i}^{k}\right)}{\partial \Delta G_{i}^{k}} \approx-\lambda^{*} ; \\
\frac{\partial P^{*}\left(\bar{G}_{i}^{k}+\Delta G_{i}^{k}, \bar{h}_{i}^{k}, \bar{g}_{i}^{k}+\Delta g_{i}^{k}\right)}{\partial \Delta h_{i}^{j}} \approx-\mu_{i}^{k *} ; \\
\frac{\partial P^{*}\left(\bar{G}_{i}^{k}+\Delta G_{i}^{k}, \bar{h}_{i}^{k}+\Delta h_{i}^{j}, \bar{g}_{i}^{k}\right)}{\partial \Delta g_{i}^{k}} \approx-\mu_{i}^{k *} .
\end{gathered}
$$

According to (34) and (35), we have the following expression

$$
\begin{aligned}
P_{\Delta}= & P^{*}\left(\bar{G}_{i}^{k}+\Delta G_{i}^{k}, \bar{h}_{i}^{k}+\Delta h_{i}^{j}, \bar{g}_{i}^{k}+\Delta g_{i}^{k}\right) \\
& -P^{*}\left(\bar{G}_{i}^{k}, \bar{h}_{i}^{k}, \bar{g}_{i}^{k}\right) \\
\approx & \sum_{i=1}^{M} \lambda^{*} \delta_{i}+\sum_{i=1}^{M} \sum_{k=1}^{N}\left(\mu_{i}^{k *} \epsilon_{i}^{k}+\mu_{i}^{k *} \omega_{i}^{k}\right) .
\end{aligned}
$$

\section{Abbreviations}

CSI: Channel state information; FBS(s): Femtocell base station(s); FU(s): Femtocell user(s); FU-Rx: FU-Receiver; FU-Tx: FU-Transmitter; HetNet(s): Heterogeneous network(s); KKT: Karush-Kuhn-Tucker; MBS: Macrocell base station; MU(s): Macrocell user(s); MU-Rx: MU-Receiver; MU-Tx: MU-Transmitter; OFDM: Orthogonal frequency-division multiplexing; OFDMA: Orthogonal frequency-division multiple access; PA: Power allocation; QoS: Quality of service; RPA: Resource power allocation; SINR: Signal-to-interference-plus-noise ratio; SIP: Semi-infinite programming; UE: User equipment

\section{Funding}

This work was supported by the National Natural Science Foundation of China (grant nos. 61601071 and 61301124), the Scientific and Technological Research Program of Chongqing Municipal Education Commission (grant no. KJ1600412), the Municipal Natural Science Foundation of Chongqing (grant nos. CSTC2016 and JCYJA2197), the Seventeenth Open Foundation of State Key Lab of Integrated Services Networks of Xidian University (grant no. ISN17-01), and the Dr. Startup Founds of Chongqing University of Posts and Telecommunications (grant no. A2016-12).

\section{Authors' contributions}

YJ contributed in the conception of the study and design of the study. Furthermore, YJ and XL carried out the simulation together. XL wrote the manuscript and completed the performance analysis of our proposed algorithm with YJ's help. YC and GQ helped to check and revise the manuscript. All authors read and approved the final manuscript.

\section{Competing interests}

The authors declare that they have no competing interests.

\section{Publisher's Note}

Springer Nature remains neutral with regard to jurisdictional claims in published maps and institutional affiliations.

Received: 15 November 2017 Accepted: 28 August 2018 Published online: 18 September 2018

\section{References}

1. D. De, A. Mukherjee, Group handoff management in low power microcell-femtocell network. Digit. Commun. Netw. 3(1), 55-65 (2017)

2. Y. Xu, Y. Hu, Q. Chen, R. Chai, G. Li, in Wireless Communications and NETWORKING Conference. Distributed resource allocation for cognitive HetNets with cross-tier interference constraint (IEEE, San Francisco, 2017), pp. 1-6

3. V. N. Ha, L. B. Le, Fair resource allocation for OFDMA femtocell networks with macrocell protection. IEEE Trans. Veh. Technol. 63(3), 1388-1401 (2014)

4. H. Zhang, D. Jiang, F. Li, K. Liu, H. Song, H. Dai, Cluster-based resource allocation for spectrum-sharing femtocell networks. IEEE Access. 4(99), 8643-8656 (2017)

5. C. T. Do, D. N. M. Dang, T. Leanh, N. H. Tran, R. Haw, C. S. Hong, in The International Conference on Information NETWORKING. Power control under QoS and interference constraint in femtocell cognitive networks (IEEE, Phuket, 2014), pp. 292-297

6. Y. Xu, X. Zhao, Y. C. Liang, Robust power control and beamforming in cognitive radio networks: a survey. IEEE Commun. Surv. Tutor. 17(4), 1834-1857 (2015) 
7. Z. Liu, P. Zhang, X. Guan, X. Li, Robust power control for femtocell networks with imperfect channel state information. let Commun. 10(8), 882-890 (2016)

8. L. Gao, Z. Liu, K. Ma, Y. Yao, in Control Conference. Robust power control for QoS guarantee in two-tier femtocell networks (IEEE, Chengdu, 2016), pp. 8397-8401

9. Z. Liu, J. Wang, Y. Xia, H. Yang, Robust optimisation of power control for femtocell networks. let Signal Process. 7(5), 360-367 (2013)

10. H. Yuan, J. Ma, H. Yang, Z. Liu, X. Guan, in Control Conference. Robust power allocation and price-based interference management in two-tier femtocell networks (IEEE, Hangzhou, 2015), pp. 6483-6488

11. Y. Zhang, S. Wang, Resource allocation for cognitive radio-enabled femtocell networks with imperfect spectrum sensing and channel uncertainty. IEEE Trans. Veh. Technol. 65(9), 7719-7728 (2016)

12. Z. Liu, S. Li, K. Ma, X. Guan, X. Li, Robust power allocation based on hierarchical game with consideration of different user requirements in two-tier femtocell networks. Comput. Netw. 122, 179-190 (2017)

13. Y. Xu, Y. Hu, Q. Chen, T. Song, R. Lai, in IEEE International Conference on Communications. Robust resource allocation for multi-tier cognitive heterogeneous networks (IEEE, Paris, 2017), pp. 1-6

14. E. Vaezpour, M. Dehghan, H. Yousefi'Zadeh, Robust distributed resource allocation in OFDMA femtocell networks. Comput. Commun. 109, 1-12 (2017)

15. Z. Liu, P. Zhang, X. Guan, H. Yang, Robust power control for femtocell networks under outage-based QoS constraints. Comput. Netw. 102(C), 145-156 (2016)

16. Z. Liu, J. Wang, Y. Xia, R. Fan, H. Jiang, H. Yang, Power allocation robust to time-varying wireless channels in femtocell networks. IEEE Trans. Veh. Technol. 65(4), 2806-2815 (2016)

17. Y. Xu, X. Zhao, Robust power control for underlay cognitive radio networks under probabilistic quality of service and interference constraints. Commun. let. 8(18), 3333-3340 (2014)

18. S. Parsaeefard, A. R. Sharafat, Robust distributed power control in cognitive radio networks. IEEE Trans. Mob. Comput. 12(4), 609-620 (2013)

19. Y. Xing, C. N. Mathur, M. A. Haleem, R. Chandramouli, K. P. Subbalakshmi, Dynamic spectrum access with QoS and interference temperature constraints. IEEE Trans. Mob. Comput. 6(4), 423-433 (2007)

20. S. Sun, J. Di, W. Ni, in International Conference on Wireless Communications and Signal Processing. Distributed power control based on convex optimization in cognitive radio networks (IEEE, Suzhou, 2010), pp. 1-6

21. K. Yang, Y. Wu, J. Huang, X. Wang, in INFOCOM 2008. the Conference on Computer Communications. IEEE. Distributed robust optimization for communication networks (IEEE, Phoenix, 2008), pp. 1157-1165

22. Z. Zhu, J. Peng, P. Yao, F. Jiang, K. Lin, in 2014 IEEE International Conference on Communications. A worst-case robust distributed power allocation scheme for OFDM-based cognitive radio networks (IEEE, Sydney, 2014), pp. 1489-1494

23. A. J. G. Anandkumar, A. Anandkumar, S. Lambotharan, J. A. Chambers, Robust rate maximization game under bounded channel uncertainty. IEEE Trans. Veh. Technol. 60(9), 4471-4486 (2011)

24. A. Ben-Tal, A. Nemirovski, Robust convex optimization. Math. Oper. Res. 23(4), 769-805 (1998)

25. L. Zhu, X. Zhao, Y. Xu, in International Conference on Computer, Information and Telecommunication Systems. Robust power control for cognitive radio networks under spectrum sensing errors (IEEE, Kunming, 2016), pp. 1-5

26. P. Setoodeh, S. Haykin, Robust transmit power control for cognitive radio. Proc. IEEE. 97(5), 915-939 (2009)

27. D. Bertsimas, M. Sim, The price of robustness. Oper. Res. 52(1), 35-53 (2004)

28. Y. Xu, X. Zhao, Robust adaptive power control for cognitive radio networks. Signal Process. let. 10(1), 19-27 (2016)

29. M. Hasan, E. Hossain, I. K. Dong, Resource allocation under channel uncertainties for relay-aided device-to-device communication underlaying LTE—a cellular networks. IEEE Trans. Wirel. Commun. 13(4), 2322-2338 (2014)

\section{Submit your manuscript to a SpringerOpen ${ }^{\circ}$ journal and benefit from:}

- Convenient online submission

- Rigorous peer review

- Open access: articles freely available online

- High visibility within the field

- Retaining the copyright to your article

Submit your next manuscript at $>$ springeropen.com 